\title{
Innovación tecnológica en el proceso de obtención de información, usando realidad aumentada en una universidad en Bolivia
}

\author{
Technological innovation in the process of obtaining information, using \\ augmented reality in a university in Bolivia
}

Jahir Elvis Zambrana Ayala, jairzinho7zam@gmail.com

Universidad Autónoma Juan Misael Saracho, Bolivia

\section{RESUMEN}

El presente estudio tuvo como propósito constituir un proyecto de innovación tecnológica manteniendo como objetivo principal sociabilizar e informar de una manera novedosa, todas las carreras que existen dentro de la Facultad de Ciencias y Tecnología de la Universidad Autónoma Juan Misael Saracho en Bolivia. La metodología de diseño de hipermedia orientada a objetos (OOHDM), fue la que se utilizó para ofrecer un marco adaptable a las condiciones del proyecto. El desarrollo de la aplicación se ha realizado con el Motor de Juegos Unity3D, SDK Android y Vuforia como SDK principal para las librerías de Realidad Aumentada. Como resultados se desarrolló una aplicación móvil Android que sirve como una herramienta de obtención de información accesible y atractiva utilizando la tecnología de Realidad Aumentada. La investigación estuvo enfocada para los estudiantes bachilleres de Tarija que se encuentren finalizando sus estudios de bachillerato. Para finalizar El estudio que se llevó a cabo encontró alternativas tecnológicas para un mejor proceso de obtención de información de las carreras de la FCYT, por consiguiente se consideró un éxito la elección del uso y aplicación de la tecnología de Realidad Aumentada.

Palabras clave: realidad aumentada; aplicación; socialización

\begin{abstract}
The purpose of this study was to establish a technological innovation project with the main goal of socializing and informing in a novel way all the careers that exist within the Faculty of Sciences and Technology of the Juan Misael Saracho Autonomous University in Bolivia. The object-oriented hypermedia design methodology (OOHDM) was the one that was used to offer an adaptable framework to the conditions of the project. The development of the application has been made with the Unity3D Game Engine, Android SDK and Vuforia as the main SDK for the libraries of Augmented Reality. As results, an Android mobile application was developed that serves as a tool for obtaining accessible and attractive information using Augmented Reality technology. The investigation was focused for the high school students of Tarija that are finishing their studies of baccalaureate. To conclude the study that was carried out found technological alternatives for a better process of obtaining information about the careers of the FCYT, therefore the choice of the use and application of the Augmented Reality technology was considered a success.
\end{abstract}

Key words: augmented reality; application; socialization 
INTRODUCCIÓN

En la actualidad día a día existen muchas herramientas tecnológicas, las cuales son usadas en distintos ámbitos para diferentes actividades, una de ellas es la Realidad Aumentada, herramienta potencial que se está abriendo espacio a pasos agigantados en el mundo actual mas no así en Bolivia.

La Realidad Aumentada (AR) es el término que se usa para definir una visión directa o indirecta de un entorno físico del mundo real, Tom Caudell 1992 cuyos elementos se combinan con elementos virtuales para la creación de una realidad mixta de tiempo real. Por lo que entonces la Realidad Aumentada es una combinación del mundo real con elementos virtuales en tiempo real, todo esto a través de un dispositivo tecnológico.

El propósito de esta investigación fue realización de una Aplicación de información general sobre la Facultad de Ciencias y Tecnología de la Universidad Autónoma Juan Misael Saracho usando la tecnología emergente de Realidad Aumentada.

Lo que lleva a identificar que le objetivo principal de este estudio fue atraer y motivar a los Bachilleres a decantarse por la Universidad Autónoma Juan Misael Saracho y su facultad de Ciencia y Tecnología (FCYT)

Por ello esta investigación expone parte de lo que fue el desarrollo de la creación de una Aplicación de Realidad Aumentada dirigida a los Bachilleres de las diferentes promociones de Tarija, Bolivia donde se obtenga Información acerca de las Carreras de la FCYT, por medio de la creación de datos virtuales y menús con informaciones como: Perfil del estudiante, misión, visión, malla curricular, modo de avance, Infraestructura, etc. Todo esto acompañado de la experiencia de la tecnología de Realidad Aumentada.

\section{Descripción y fundamentación del proyecto}

La Realidad Aumentada es una Herramienta Tecnológica de inmensas aplicaciones como lo es: Turismo, Publicidad, Educación, Industria, Entretenimiento, entre otras.

Este tipo de tecnologías tiene la capacidad intrínseca de causar curiosidad y atención, además de llegar a los usuarios de una manera diferente ya que modifica el modo de generar el aprendizaje.

La Tecnología de Realidad Aumentada se puede manifestar de diferentes maneras debido a su software, ya sea con Global Positioning System (GPS), sensores de entorno o con marcadores que serían la llave activadora que permite la visualización de la realidad aumentada.

Es por eso que se decide tomar esta tecnología para adaptarla en un aplicativo móvil el cual contenga información de las carreras de la Facultad de Ciencias y Tecnología. Hoy en día existen varias formas de conseguir información sobre estas carreras, como ser:

Universidad Abierta, es un evento general que realiza la UAJMS, dirigido a los estudiantes de la promoción de los diferentes colegios de Tarija, con motivo de dar a conocer las instalaciones $y$ brindar información sobre las diferentes carreras de las cuales consta la Institución, además de la Facultad de Ciencias y Tecnología con todas sus carreras también se hacen presente en este evento.

Canal Universitario, que es un medio de comunicación de la UAJMS en donde se difunden Spot Publicitarios de las carreras e información vinculante a la Institución, la misma se creó como brazo fundamental de la Universidad, para coadyuvar con los programas de carácter educativo, cultural, científico y recreativo. 
Portal Universitario, es la página oficial de la universidad en la cual se encuentra información y noticias sobre las diferentes facultades, fechas, costos, etc., pero que lastimosamente no es una opción utilizada por el que el estudiante actual se decante.

Entonces la opción por la que más optan los bachilleres, es apersonándose al rectorado o a las distintas facultades para obtener algún tipo de información, algunas veces se procede a derivarlos a otra oficina donde se puede adquirir una guía del estudiante, pero el costo monetario muchas veces lo vuelve un material privativo, la última gestión incluso no se encontró disponible este material ni para la venta.

Por lo consiguiente el bachiller muchas veces no goza del tiempo suficiente para escoger su vocación por la poca información que se le brinda, porque no consigue la información de manera fácil y cuando lo logra hay un descontento por la información mostrada de manera global, que existe en la guía o la página de la universidad, ahí empiezan las dudas sobre la carrera a inclinarse, otro factor es el estrecho tiempo que suele comprender entre salir Bachiller y entrar a los cursos Preuniversitarios o exámenes directos de nuestra Universidad, por lo que se ven envueltos en tomar una decisión rápida, pero a la vez confusa.

Lo que pretende este Proyecto de Realidad Aumentada es el de servir en el mejoramiento de la experiencia de los Bachilleres al obtener información, en relación a nuestra Casa Superior de Estudios, utilizando una visualización más atractiva e innovadora para incentivar la curiosidad y atraer a un mayor número de futuros estudiantes a la FCYT de la UAJMS.

MATERIALES Y METODOS

Carrillo (2009) dice que la construcción de grandes aplicaciones hipermedia es extremadamente difícil, por otro lado no existe una metodología que se adapte perfectamente a este tipo de software, tentando a los desarrolladores a la omisión del diseño estructural de la aplicación. Sin embargo, este planteamiento traería nefastos resultados en el momento de los mantenimientos del software.

Es por ello, que para la elaboración de este estudio se usaron componentes y dispositivos tecnológicos que permitieron la creación de una adecuada programación de tareas antes de la construcción de la aplicación. Para lograr esto, se utilizaron metodologías de desarrollo con modelos y estructuras formales de diseño e implementación especialmente orientadas a un software hipermedia.

Habitualmente el desarrollo de Sistemas Hipermedia suele hacerse directamente a nivel de implementación, descuidándose el importante proceso previo de análisis y diseño de los aspectos de la navegación e interfaz sin embargo, en los últimos años existe una tendencia a considerar el desarrollo hipermedia con un enfoque de proceso de ingeniería del software, por lo que ya se han propuesto diferentes metodologías, como:

- HDM (Modelo de Diseño de Hipertexto)

- EORM (Modelo de relación de objeto mejorado)

- RMM (Metodología de Gestión de Relaciones)

- OOHDM (Método de diseño de hipermedia orientado a objetos).

(Edured)

En el presente trabajo adoptó el Modelo de Hipermedia Orientado a Objetos como metodología de Ingeniería de Software y para el componente de Socialización utilizó la metodología de estrategias discursivas esta será la que se mostrara en los resultados. 
RESULTADOS

Esta investigación presentas las actividades que se llevaron a cabo para la realización de la socialización para difundir la Tecnología (Realidad Aumentada) y así mejorar los conocimientos sobre esta área, para ello fueron implementadas estrategias de socialización que permitieron la difusión y divulgación a través de los medios televisivos y radiales propios de la institución académica Universidad Autónoma Juan Misael Saracho ubicada en Tarija, Bolivia.

La socialización es un proceso de influencia entre una persona $y$ sus semejantes, se observa no solo en las distintas etapas entre la infancia y la vejez, sino también en personas que cambian de una cultura a otra, o de un status social a otro, o de una ocupación a otra. Por lo que Yubero, (2005) que plantea que:

\begin{abstract}
"Puede decirse que el ser humano desde que nace es un ser social, destinado a vivir en un mundo social y que, para ello, necesita la ayuda de los demás. Ese ser social, además, se va haciendo poco a poco a través de la interacción con los otros, en un proceso continuo de socialización." (s/p).
\end{abstract}

La socialización es vista por los sociólogos como el proceso mediante el cual se inculca la cultura a los miembros de la sociedad, a través de él, la cultura se va transmitiendo de generación en generación, los individuos aprenden conocimientos específicos, desarrollan sus potencialidades y habilidades necesarias para la participación adecuada en la vida social y se adaptan a las formas de comportamiento organizado característico de su sociedad. (Yubero, 2005)

La socialización a la que se somete a las generaciones más jóvenes es el resultado de un proceso educativo que planifica y dicta la sociedad.

El proceso de socialización es de naturaleza esencialmente interactiva, implicando un aprendizaje por parte de la persona socializada, quien va a ser objeto, en función de su papel moldeador, de diferentes cambios. Hemos de considerar que la socialización supone un proceso de adaptación a la sociedad que necesariamente es educativo, ya que implica el aprendizaje de una serie de contenidos que la sociedad ha preparado para tal fin.

La Aplicación fue exportada a la plataforma Android, y cuenta con información considerable e importante de las diferentes carreras de FCYT.

El estudio encontró lugar para que los estudiantes bachilleres pudiesen disfrutar de esta experiencia con el uso de una tarjeta o marcador que se le obsequiará al momento de preguntar por las Carreras de la FCYT, las tarjetas entregadas tendrán información alojada en ellas, el cual los estudiantes deben escanear para posteriormente descargar un software en sus dispositivos, una vez descargado lo instalaran en su dispositivo móvil.

\section{Propósito de la socialización de la Tecnología Realidad Aumentada}

A través de esta socialización se pretendió alcanzar los siguientes objetivos:

- Dar a conocer la importancia de las nuevas tecnologías en laeducación.

- Dar a conocer la existencia de la aplicación a los estudiantes.

Existen diversas formas de socializar un producto, ya sea por los medios de comunicación, por las redes sociales, por agentes de socialización, entre otros.

Para la socialización de la aplicación como se ha indicado en la matriz de marco 
lógico del estudio se realizó la visita a distintos colegios en los cuales se desarrolló una explicación con respecto al tema Realidad Aumentada y posteriormente una demostración de la aplicación.

En los colegios seleccionados para la socialización, los estudiantes se notaron interesados durante una demostración realizada en el evento de Universidad Abierta, evento que se hace a nivel general para promocionar todas las carreras que existen la UAJMS.

Se logró el acceso a las instituciones escolares por parte del director del Departamento de Informática y Sistemas él fue el encargado en tramitar y facilitar el ingreso y publicidad de la aplicación.

\section{Metodología de Estrategias Discursivas}

La metodología de enseñanza adoptada para el componente de socialización fue la de metodología de Estrategias Discursivas.

El termino Estrategias Discursivas refiere a los diferentes medios en que usa un hablante para adquirir, comprender, producir y reproducir el habla, para evaluar de manera eficiente el significado, las funciones lingüísticas, interacciónales, sociales, pragmáticas y psicológicas de los enunciados en el discurso. (Mijangos, 2017).

Fueron adaptados dos tipos de esta metodología, como lo es Método Discursivo Descriptivo y Expositivo.

El discurso Descriptivo está centrado en la explicación o enumeración de las propiedades o características de una persona, objeto, lugar o situación. (Correa, 2015).

El discurso Expositivo se caracteriza porque en el predomina la finalidad del traspaso informático ala receptor. Es decir, a través del discurso expositivo se intercambia información. El emisor es un sujeto que conoce un tema, mientras que el receptor es el individuo que será informado sobre ese contenido (Correa, 2015).

\section{Programa}

- Breve explicación de Realidad Aumentada

- Diferencia con tecnologías similares como la Realidad Virtual

- Usos y Aplicaciones en la actualidad

- Conclusiones Personales

\section{Desarrollo del Proceso de Socialización}

Para el desarrollo de nuestra socialización se llevaron a cabo visitas en diversas Unidades Educativas, las mismas se realizaron en los siguientes colegios:

- Unidad Educativa "Gral. Manuel Belgrano"

- Unidad Educativa "Maria Laura Justiniano"

- Unidad Educativa "Carmen Mealla"

- Unidad Educativa "Tercera Orden Franciscana"

- Unidad Educativa "San Roque"

- Unidad Educativa “UBEET"

En cada institución se pidió reunirse con el director, en algunos casos en su mayoría solo se logró hablar con los profesores o la secretaria del establecimiento, se les dio a conocer la intención o propósito para con los estudiantes de la promoción, se obtuvieron distintas respuestas por parte de los colegios al estar en su mayoría en actividades finales de la etapa escolar, por consiguiente hubo complicaciones para que pudiesen aceptar la petición.

Sin embargo hubo dos colegios que nos brindaron el apoyo y la confianza para realizar la socialización y completar el objetivo.

La primera semana se enfocó en visitar los colegios que quisieran que en la 
semana posterior se realice la socialización, en la segunda semana la misma se realizó cumpliendo el programa y tuvo una duración aproximada de 30 minutos por cada exposición en los colegios.

Los 2 colegios con los cuales se realizó el componente de socialización de este proyecto fueron:

- Unidad Educativa "Gral. Manuel Belgrano"

- Unidad Educativa "San Roque"

Estos colegios cuentan cada uno con 2 grados " $A$ " y "B", que en total serian 4 cursos para realizar la socialización. Pero en el colegio Gral. Belgrano solo se pudo socializar a un curso debido a que el otro se encontraba constantemente en evaluación. Entonces:

San Roque $=2$ cursos de promoción, socialización (2 cursos) Gral. Belgrano $=2$ cursos de promoción, socialización (1 curso)

Después de culminar la socialización se les facilito a los estudiantes un enlace para acceder a un formulario de Google que contenía una encuesta virtual calificativa sobre la difusión de la tecnología realizada, con la finalidad de que estos evaluaran la aplicación y la socialización que se había llevado a cabo en estas instituciones.

El proceso de socializar un trabajo es muy gratificante, los estudiantes demostraron interés y empatía por el trabajo realizado, se comprobó también que la asimilación de cualquier tipo de información siempre se potencia con nuevas formas de mostrarla o visualizarla, incluso el interés de los profesores se hizo visible al ser partícipes también de la socialización.

Así mismo se recomienda hacer este tipo de socializaciones en fechas menos ajetreadas para los colegios, ya que se entiende que hubo el deseo de colaborar con la causa, pero los tiempos impedían su ejecución.

La elección de la tecnología de Realidad Aumentada para fusionarla en una aplicación informativa fue una decisión correcta y se refleja en los resultados obtenidos en la encuesta realizada.

\section{Medios Verificables del Componente 2}

Los medios verificables del Componente 2 son:

- Fotos de las socializaciones realizadas en la Universidad Abierta

- Fotos de socialización realizada dentro los colegios.

- Carta de socialización realizada, firmada por el/la profesor(a) del curso.

- Resultados de la encuesta realizada en los cursos visitados, realizada por el Director del Proyecto. 

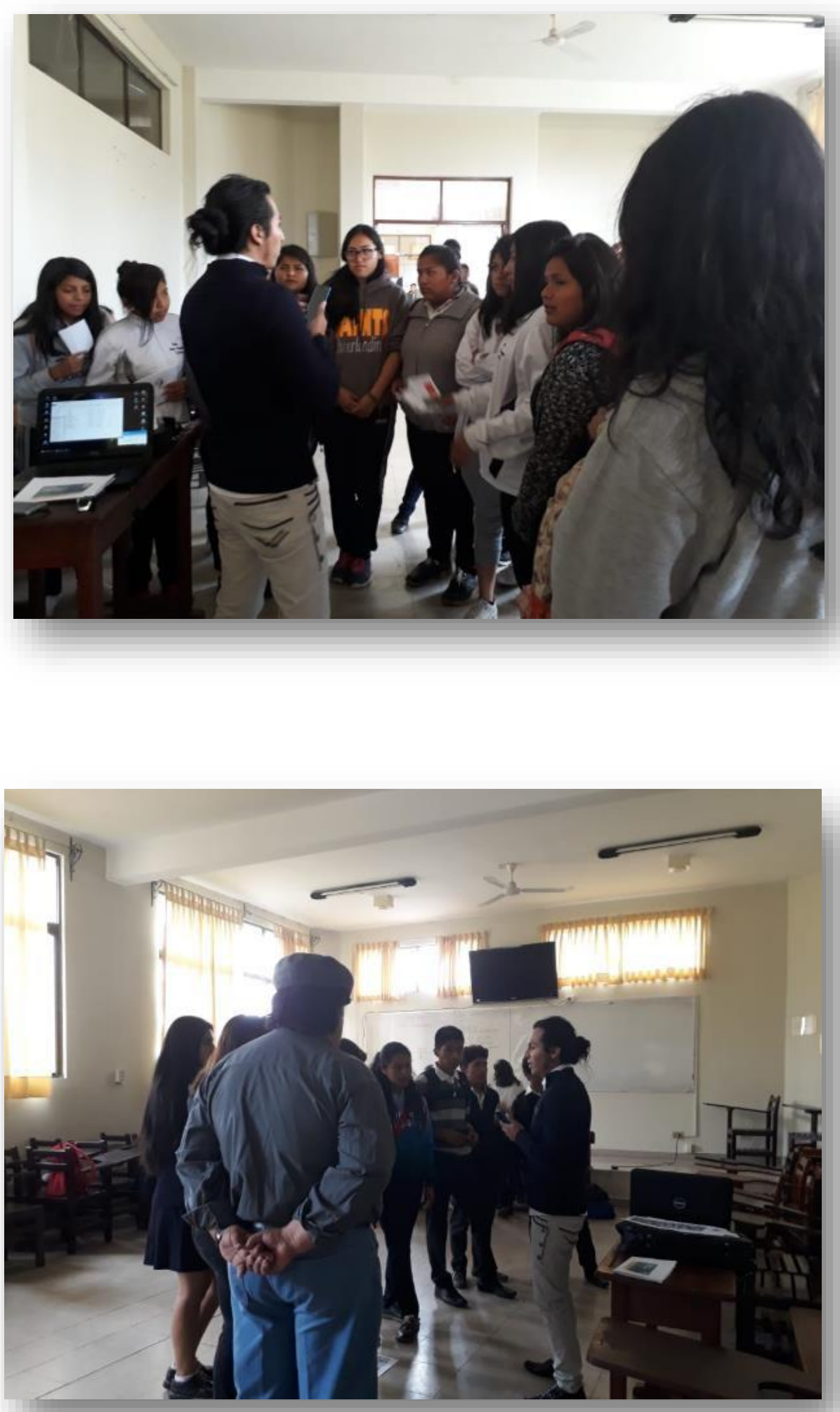

Imagen 1. Fotos de las Socialización en el evento de Universidad Abierta 


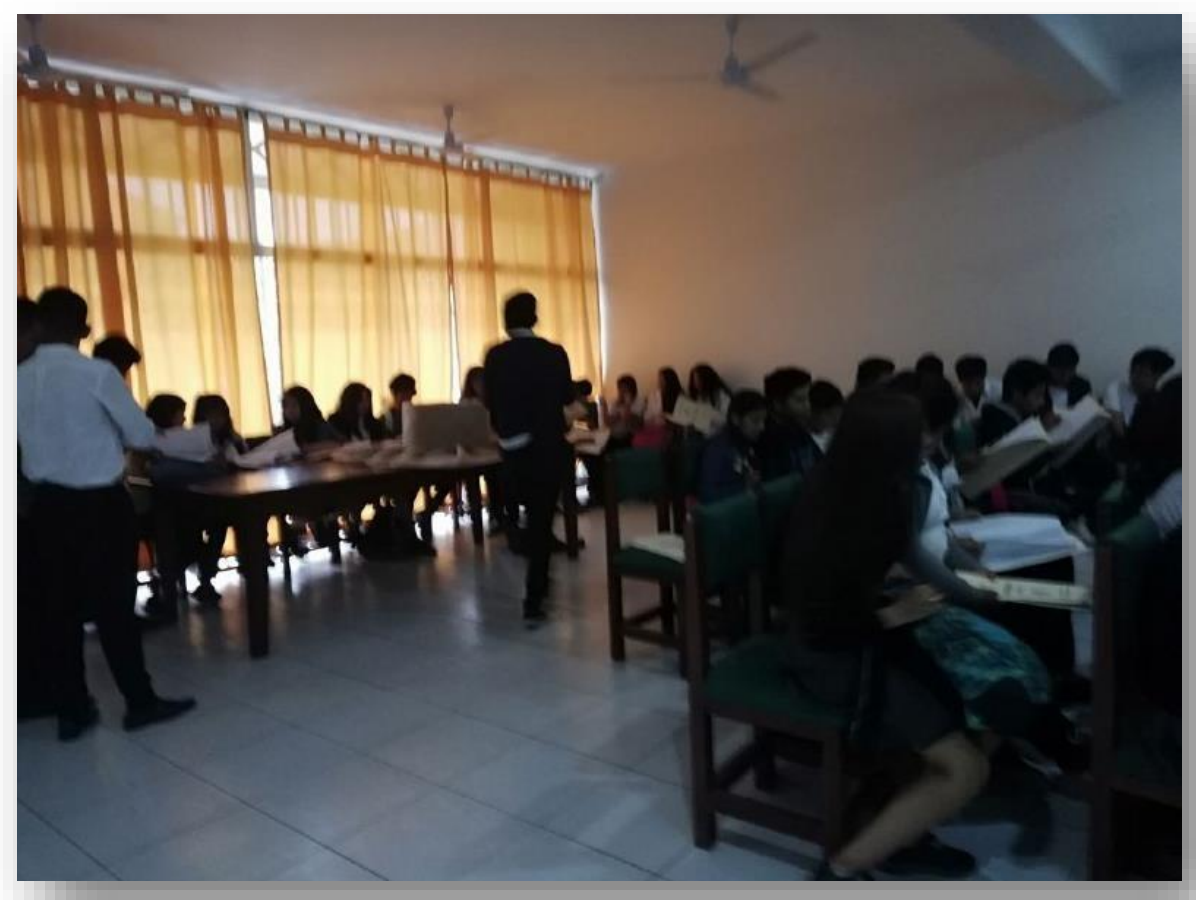

Imagen 2. Fotos Socialización realizada en Colegios Unidad Educativa "San Roque"

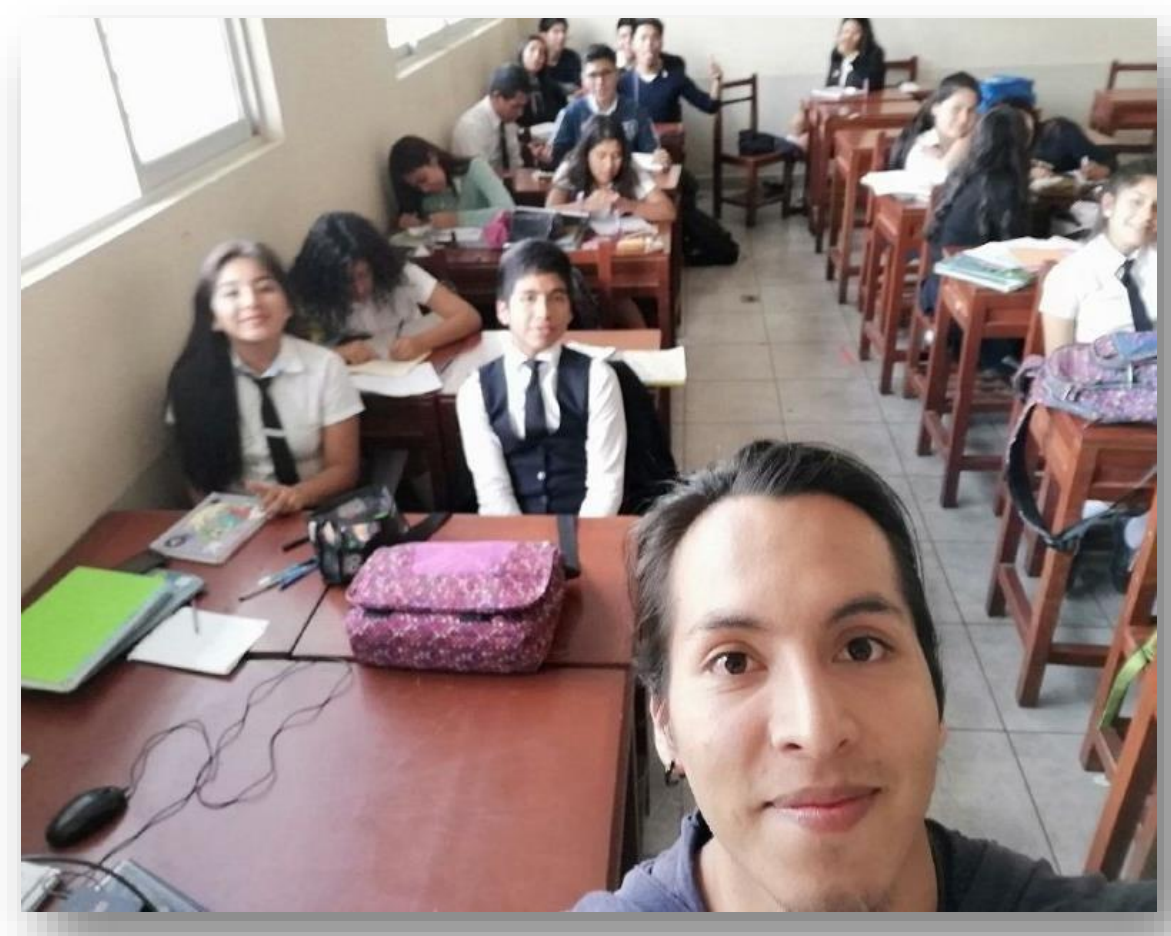

Imagen 3. Unidad Educativa "Manuel Belgrano 


\section{Resultados de la Encuesta}

Se realizó una encuesta virtual a los estudiantes una vez que se completó la socialización, esto se realizó con ayuda de la herramienta de Google Form.

Los resultados obtenidos se muestran a continuación:

1. Te pareció interesante la tecnología mostrada en clase?

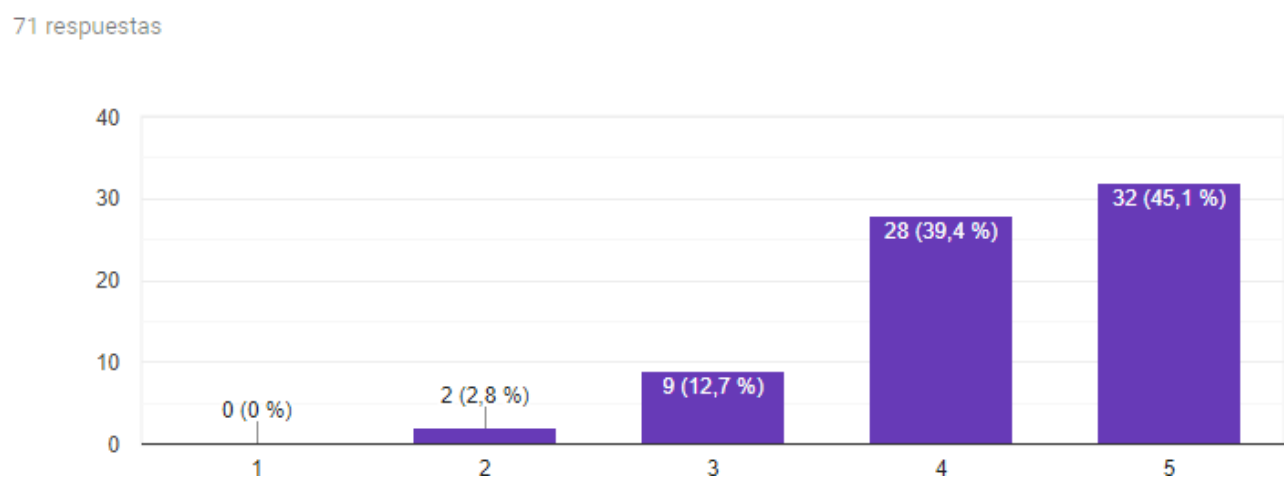

2. Entendiste el proceso básico del funcionamiento de esta tecnología?
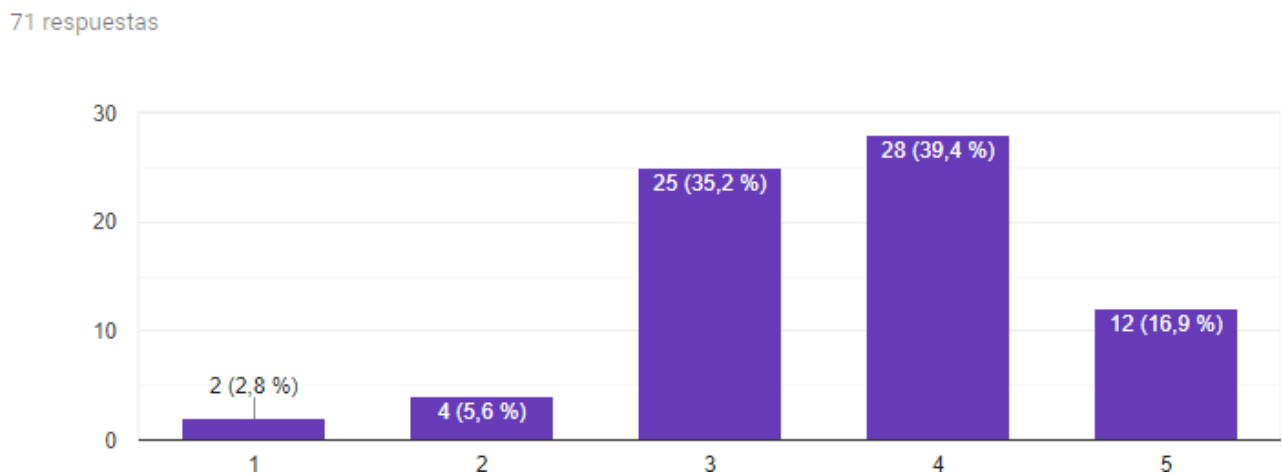

3. Crees que esta tecnología cambia la manera de ver la información?

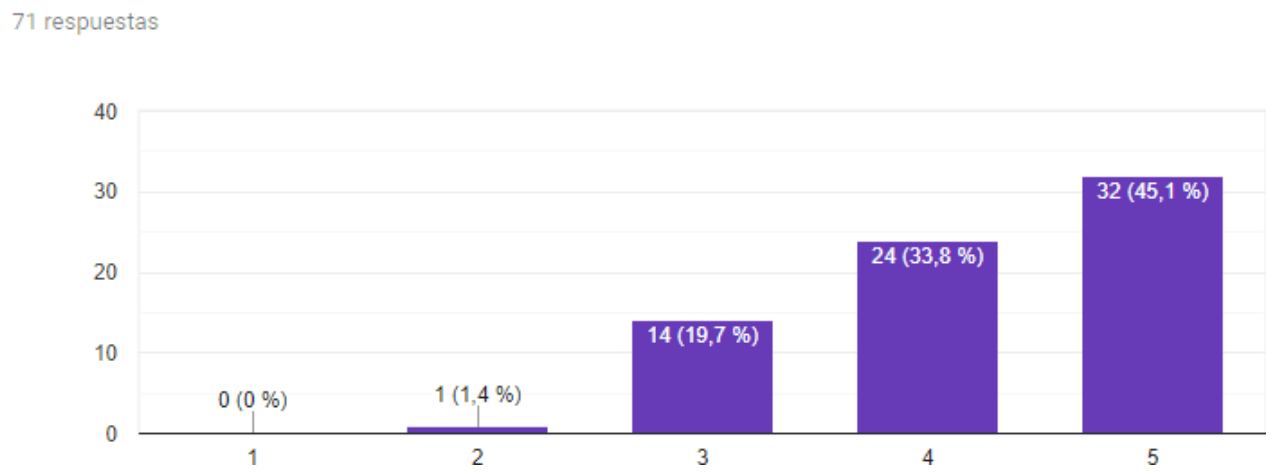


4. Sobre la aplicación mostrada al final de la exposición ¿Qué te pareció?

71 respuestas

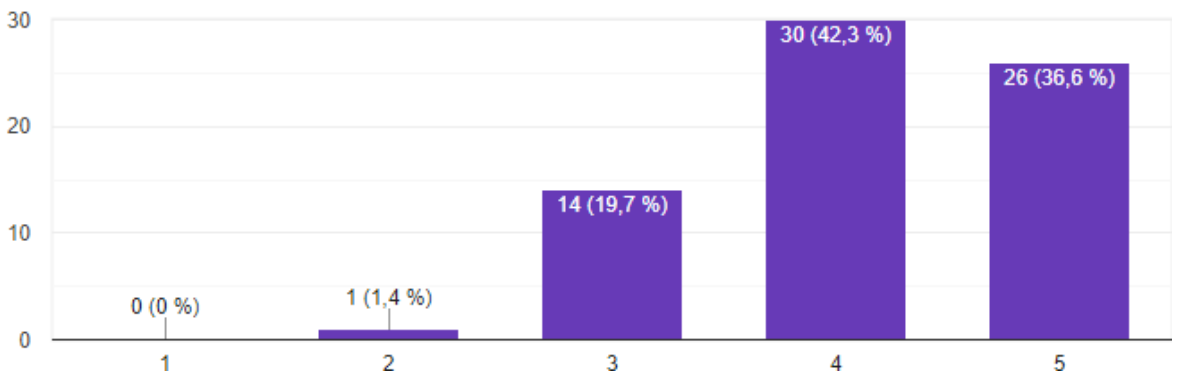

5. Piensas que tienes un poco más de conocimiento sobre esta tecnología y sobre las carreras de la FCYT mostradas en clase?

71 respuestas

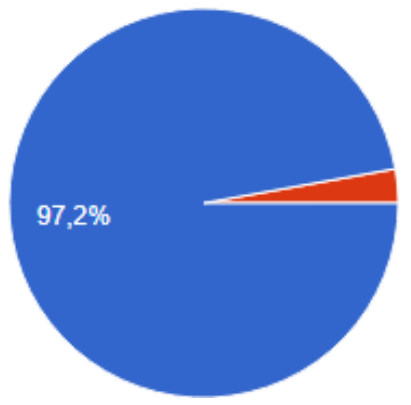

Con la evaluación aplicada en las instituciones participantes queda por sentado que la socialización de la tecnología de Realidad Aumentada cumplió con la aceptación de los estudiantes y se cumplió los objetivos trazados.

\section{CONCLUSIONES}

El estudio que se llevó a cabo encontró alternativas tecnológicas para un mejor proceso de obtención de información de las carreras de la FCYT, por consiguiente se consideró un éxito la elección del uso y aplicación de la tecnología de Realidad Aumentada, así como el desarrollo de la aplicación móvil para Android que encapsulo toda la información precisa que los bachilleres necesitan. Donde todos los requisitos establecidos se lograron cumplir a cabalidad y a la finalización del mismo, se pudo evidenciar el despliegue de un apk funcional.

Por otra parte, la socialización realizada a los dos colegios que nos abrieron las puertas de sus aulas, resulto positiva ya que la tecnología fue aceptada y acogida por los estudiantes participantes en las aulas como en los resultados de la encuesta virtual, razón además que comprobó el acierto del estudio y generó una inyección de motivación adicional.

Definitivamente la Realidad Aumentada es y será una tecnología que seguirá abriendo espacio en nuestro entorno, lo utilizáramos o no, ofrece diversas posibilidades y a diferencia de 
otras tecnologías emergentes pasajeras, esta tecnología tiene fuertes bases de investigación, desarrollo y apoyo en el mundo.

\section{REFERENCIAS}

Caudell, T. (1992). AR: sigla utilizada para referirse a la realidad aumentada viene del inglés Augmented Reality

Correa L. (2015) "Estrategias Discursivas" [Presentación] Obtenido de: https://prezi.com/tgygrl1pvwtx/estr ategias-discursivas /

Ecured (s.f.) Metodologías para Realidad Aumentada. Obtenido de: https://www.ecured.cu/Metodologías _para_el_diseño_de_aplicaciones_multi media

Garlan D. y Shaw M. (1994). An Introduction to Software Architecture. CMU- CS-94-166 School of Computer Science Carnegie Mellon University $-844$
Pittsburgh, PA 15213-3890 Obtenido de:

https://www.cs.cmu.edu/afs/cs/proj ect/able/ftp/intro_softarch/intro_soft arch.pdf

GPS: Global Positioning System, Sistema de Posicionamiento Global

Mijangos, R., y del Carmen, A. (2017). Métodos de enseñanza. Disponible en: https://m.monografias.com/trabajos1 5/metodos-ensenanza/metodosensenanza.shtml

Ramos, A. C. (s/f). Herramienta Multimedia de apoyo a la Enseñanza de la Metodología RUP de Ingeniería del Software.

Yubero, Santiago (2005) PSICOLOGÍA SOCIAL, CULTURA Y EDUCACIÓN, coord. por Darío Páez Rovira, Itziar Fernández Sedano, Silvia Ubillos Landa, Elena Zubieta. Pág. 819 